\title{
Tele-care for emergency announcements
}

\author{
Ching-Sung Wang ${ }^{1}$, Chien-Wei Liu ${ }^{2}$, Teng-Wei Wang ${ }^{3}$ \\ ${ }^{1}$ Department of Electronic Engineering, Oriental Institute of Technology, Taipei, Taiwan, China; \\ ${ }^{2}$ Department of Information Management, St. Mary’s College, Yilan, Taiwan, China; \\ ${ }^{3}$ The Third Department of Clinical Research Institute, Peking University, Beijing, China. \\ Email: ff020@mail.oit.edu.tw
}

Received 5 June 2010; revised 21 June 2010; accepted 22 June 2010.

\begin{abstract}
The first aid and immediately help are very important in an accident. The earlier detection and treatment we do, the better prognosis and health patients have. In the senior populations, it is more important. Once seniors have an accident, not only physically injure in their body, but also mental and social ability, and may have severe sequela. Concerning about these populations, this research design a simple, practical, and portable device of real-time monitoring body activity with sphygmomanometer and pulsimeter. When an accident occurs, the signals go through mobile phone, immediately notify the remote ends and provide first time help.
\end{abstract}

Keywords: Real-Time; Monitoring; Mobile; Sphygmomanometer; Pulsimeter

\section{INTRODUCTION}

In 1991, American Heart Association suggests "The Chain of Survival” -Early Access, Early CPR, Early Defibrillation, and Early Advanced Care. It emphasizes that immediately after cardiac arrest, early CPR in the first $4 \mathrm{~min}$. and early advance care in the first $8 \mathrm{~min}$ would have about $43 \%$ success rescue rate, on the contrary, lower than $20 \%$. Cumnius, R.O.'s research points out that there is about $7-10 \%$ success rescue rate decreasing per minute delayed after patients' heart arrest [1]. In traumatic care, there is so called "golden hour" concept that means the first sixty minutes after the occurrence of multi-system trauma. It is widely believed that the victim's chances of survival are greatest if they receive definitive care in the operating room within the first hour after a severe injury (Committee on Trauma, 1993; Division of Trauma \& EMS, 1992). For the cerebrovascular accident, it also points out "golden 3 hours", giving a proper deal in the first 3 hours after the accident occurring can increase the chance of survival. Whether what kind of emergency, they focus on immediately re- sponse and properly deal, early detection and early treatment.

Accident is more important for the seniors, morbid populations and for those who have unstable life signs. According to studies, once seniors fall down, they would reduce their living activity [2,3], indirectly leveling down their life quality.

Based on people's habits, using mobile phone, this research suggests a device, real timely monitoring human body's activity with sphygmomanometer and pulsimeter, combining with mobile and Bluetooth etc. relative technology to edit a simple, practical, and suit for the current mobile phone that includes Bluetooth device. When the accident occurs, it can immediately and effectively provide relative rescuing information to remote GPS etc, expecting that is able to improve first aid effect. And, it provides senior population an effective preventing accident guarantee, enabling them to avoid decreasing their life activity caused by an accident.

\section{SYSTEM ARCHITECTURE}

This system divides into two parts, which are client side and server side respectively. Client side comprises BAD (Body Activity Detector) with sphygmomanometer and pulsimeter, GPS (Global Position System) device and mobile phone. Once BAD detected any unusual body responses, mobile phone would immediately delivers the emergent message by DTMF (Dual Tone Multi Frequency) coding with sphygmomanometer, pulsimeter and the GPS coordinates to the monitoring server [4]. The server, which includes PSTN (Public Switched Telephone Network) phone controller and a monitoring terminal, that is responsible for monitoring the client and emergency management. Figure 1 shows the system architecture.

\subsection{Client Side System}

As considered the mobility, BAD with sphygmomanometer, pulsimeter and GPS devices are separated in client side device, yet information delivery by Bluetooth. 


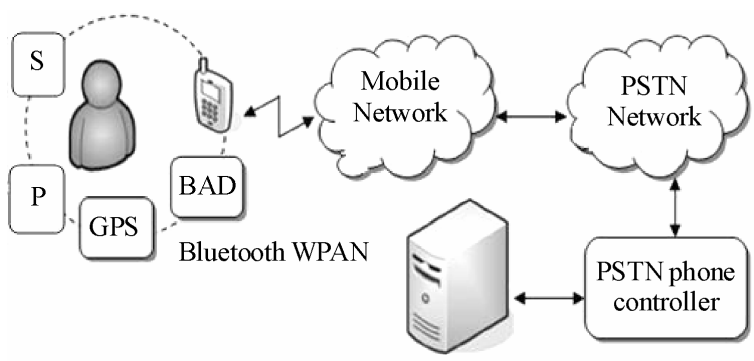

Figure 1. System architecture (P: pulsimeter; S: sphygmomanometer).

Details of BAD with sphygmomanometer, pulsimeter and GPS devices are mentioned in the following:

1) BAD: The main function is to monitoring the unusual body activity, and communicates with mobile phone by Bluetooth HFP (Hands-Free Profile) [5]. When BAD detects any unusual body responses, the emergent signals and the GPS coordinates information will be delivered to the mobile phone, and they simultaneously transport the signals to the cell phone by Pulsimeter and Sphygmomanometer, and the mobile phone sends such message to the monitoring server through the public mobile network immediately.

2) GPS Device: receiving GPS signals. GPS device could deliver GPS signals to BAD by Bluetooth SPP (Serial Port Profile) [6] every 5 seconds to keep BAD updated the latest position information.

3) Pulsimeter and Sphygmomanometer: when Pulsimeter and Sphygmomanometer receive any urgency messages from BAD, they immediately measure the urgency messages and transport the results to the cell phone by the BAD of Bluetooth. Then Pulsimeter and Sphygmomanometer transport them every 10 seconds.

\subsection{Server Side System}

Server side is constructed by PSTN phone controller and the monitoring terminal. When server side PSTN phone controller receives the incoming call from client side mobile phone, it will be forced to connect and output the sound to the monitoring terminal. The monitoring terminal reads the DTMF code and once the terminal determines the emergency signals are correct, it would send out the emergency tone and pop out the emergency screen.

\section{HARDWARE ARCHITECTURE SHORT-LISTED}

\subsection{Client Side Hardware Architecture}

Figure 2 details the block diagram of BAD hardware architecture. BAD is mainly constructed by a microprocessor, an accelerometer, Bluetooth transceiver, voice

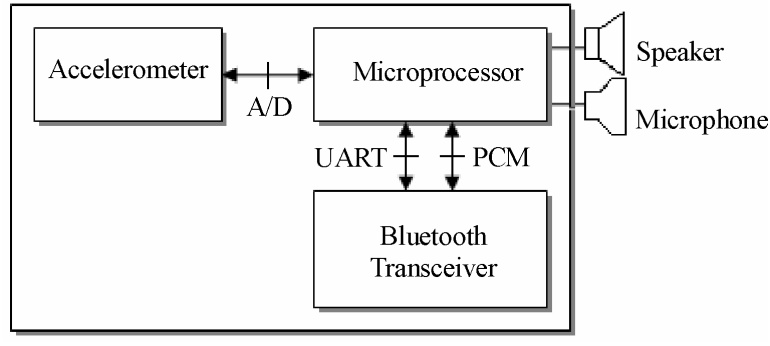

Figure 2. BAD hardware architecture.

device and other accessories. Its functions are detection of the body reaction signals, anomaly analysis, and the control of signal transfer and receive. Explanations of each device are listed below:

1) Microprocessor: SC14431 [7] is used as the controller of BAD in this research. SC14431contains a RISC (Reduced Instruction Set Computer) micro controller, DSP (Digital Signal Processor), and PCM (Pulse Code Modulation) codec. Micro controller is used for operation of Bluetooth protocol stack, and analysis of the signals of accelerometer. DSP and PCM codec are used for sound signal operation and decoding. SC14431 is made by SiTel.

2) Accelerometer: MXA2500 Dual Axis Accelerometer [8] is used as the accelerometer is this research. MXA2500 is an electro-mechanical integrate device which can be used to detect the change of acceleration. The change of acceleration is a control point of the body condition in this research. MXA2500 communicates with the microprocessor through the A/D (Analog/Digital) interface. MXA2500 is made by MEMSIC.

3) Bluetooth Transceiver: BC03 [9] is used as the baseband IC of Bluetooth transceiver, communicates with the microprocessor through UART and PCM interface. BC03 is made by CSR.

Figure 3 details the block diagram of GPS device hardware architecture. The hardware structure of GPS device is similar to BAD, which contains the microprocessor, GPS module, Bluetooth transceiver and the

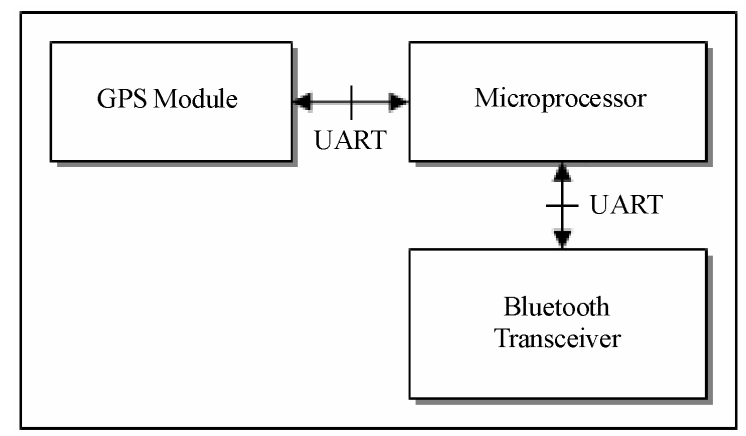

Figure 3. GPS device hardware architecture. 
accessories. Microprocessor is used for the operation of Bluetooth protocol stack and receiving of the information of GPS module. Start2 GRF2I [10] is used as the GPS module RF IC and Start2 GSP2e is used as the GPS module baseband IC, which deliver the data to the microprocessor through UART interface. Start2 GRF2i and GSP2e are made by SiRF.

Sphygmomanometer [11-14]: The sphygmomanometer includes LDO (Low Dropout Regulators) as power controller, pressure sensor, filter, amplifier circuit and accessories. The research is using the pressure sensor of SCC series which is manufactured by HoneyWell company. The pressure sensor will transmit different message according to the changing pressure. The message passes through the procedures of magnifying (magnify the micro-message form sensor), and filtering (remove noise), then all the analog messages will be sent to the microcontroller via A/D (Analog/Digital) interface. Figure 4 shows the block diagram of sphygmomanometer hardware architecture.

Pulsimeter [11-14]: The Pulsimeter includes LDO, pressure sensor, filter, amplifier circuit and accessories. The circuit is using the SCC series of HonyWell company, but it is a different sensitivity pressure sensor. Putting sensor pad on pulse of hand, the pressure sensor will convert the normal pulse beat to a larger voltage output by magnifying, fillering, comparing (transform to digital signal) then inputs it into microcontroller. Figure 5 is shown the block diagram of Pulsimeter.

\subsection{Server Side Hardware Architecture}

Figure 6 details the block diagram of server side hardware Architecture. Server side includes a PSTN phone controller and a monitoring terminal. Furthermore, PSTN

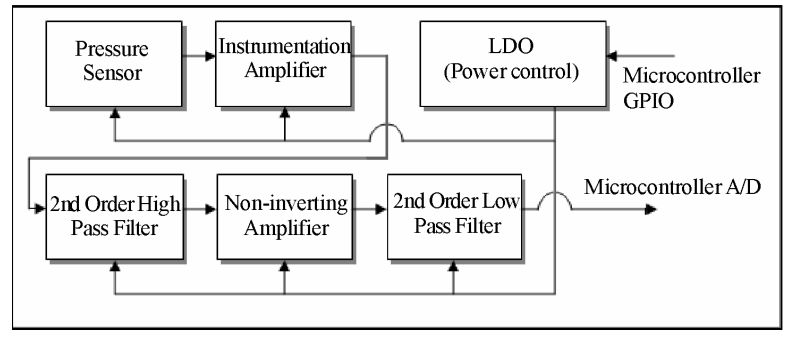

Figure 4. Sphygmomanometer hardware architecture.

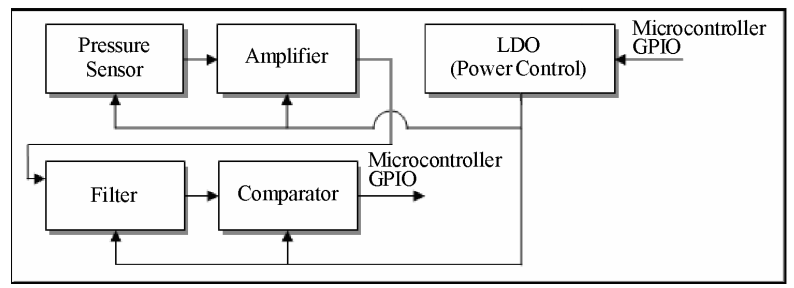

Figure 5. Pulsimeter hardware architecture.

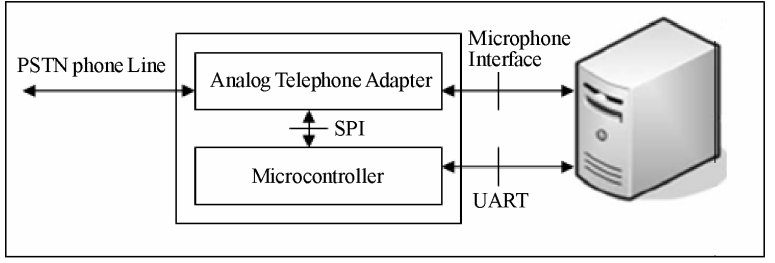

Figure 6. Server side hardware architecture.

phone controller is constructed by ATA (Analog Telephone Adapter), microcontroller, and the accessories, which takes the incoming call from the client. W681388 [15] is used as the baseband IC of ATA for ring detection and coding of signals of voice. PIC24FJ64 [16] is used as microcontroller, which receives the command of the monitoring terminal through the UART interface and controls W681388 Hook ON/OFF and voice output through SPI(Serial Peripheral Interface) interface. W681388 is made by Winbond. PIC24FJ64 is made by Microchip.

\section{WIRELESS PERSONAL AREA NETWORK BASE ON BLUETOOTH}

Bluetooth provides point to point and multipoint wireless connection according to the internet concept. Within any active communication scope, any devices are treated the same. The first one requesting communication is called master, and the passive one to accept signal is called slave. A master and one or more salves construct the Piconet of Bluetooth $[17,18]$. Due to the reason that not all the mobile phone with Bluetooth function in the current market support Serial Port Profile, the GPS device used in this research cannot connect with all the mobile phone with Bluetooth via Bluetooth function. In order to cover most of the mobile phone with Bluetooth in the market in this research and make an active Piconet, we use the Bluetooth device of BAD as master, and the Bluetooth devices of mobile phone and GPS as slave. The Bluetooth of BAD connects with the Bluetooth of mobile phone and GPS and form a PAN (Personal Area Network) upon Bluetooth. Figure 7 indicates the Bluetooth Piconet in this research.

\section{SINGAL PROCESSING}

BAD in this research contains two different modes according to different body conditions, which are body stimulation mode and body activity detection mode respectively. Here are the details below:

\subsection{Body Stress-Reaction Mode Signal Processing}

In this mode, it means the system detect the body condition has changed from a low activity level to a continuing high consumption level and it represents there is something happened [19]. Then BAD turns into the 


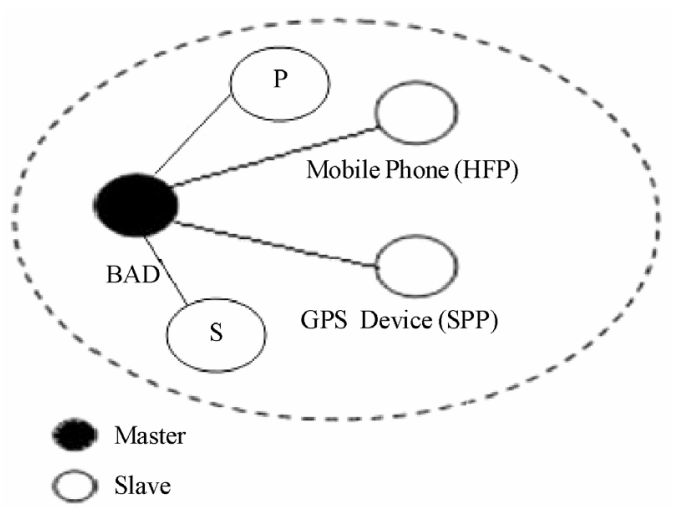

Figure 7. Client side bluetooth piconet (P: pulsimeter; S: sphygmo-manometer).

emergency processes right away. For example if the user falls down or breaks out epilepsy, the change of energy reaction would trigger the emergency task. Figure 8 indicates the sample accelerometer signal in the low activity level of body. Figure 9 indicates sample of accel-

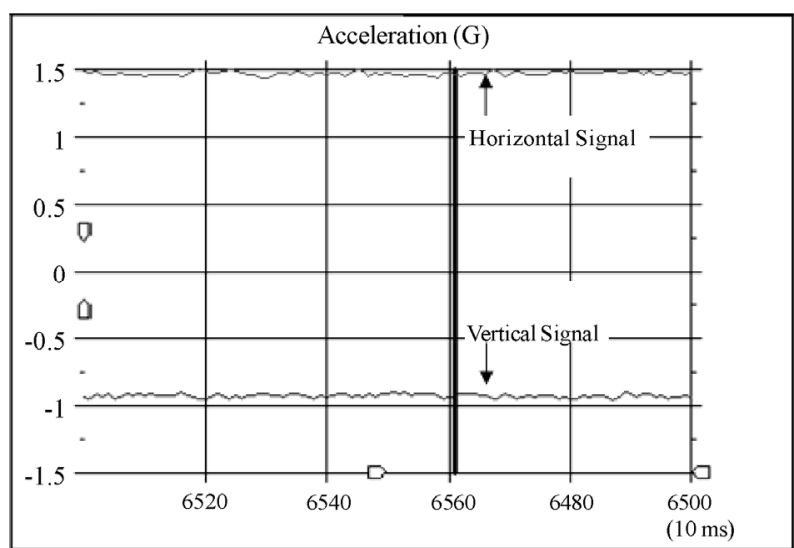

Figure 8. Sample of accelerometer signal in the low activity level of body.

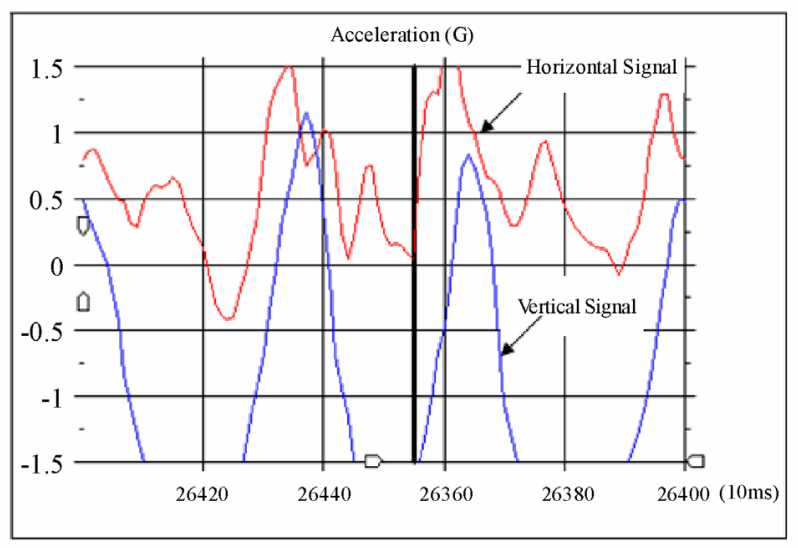

Figure 9. The sample accelerometer signal in the high activity level of body. erometer signal in the high activity level of body. Figure 10 indicates the body stress-reaction mode procedure flow chat.

Microprocessor reads the signal from accelerometer every 10 milliseconds, and pass the signal through one order passive HPF (High Pass Filter). Refer to Eq.1 [19], and then calculate the RMS (Root Mean Square). Refer to Eq.2, if the RMS is upper than the preset dynamic RMS threshold for a period of time, it means that emergency has occurred. The system would beep out the warning sound in the first place and if the user does not turn off the alarm, the system would start emergency procedure.

$$
Y(n)=R \times C \times(X(n)-X(n-1)+Y(n-1)) /(T+R \times C)
$$

HPF is used to screen out the signals over 1HZ. While the signal is over $1 \mathrm{HZ}$ it means it is not the system targeted signals. It perhaps just comes from the shaking or noises of the devices. $1 \mathrm{HZ}$ HPF RC $=0.16, \mathrm{~T}=0.01$ (100HZ) [19].

$$
Y(r m s)=\sqrt{\frac{1}{n} \sum_{i=1}^{n} Y i^{2}}
$$

RMS: The accelerometer provides absolute analog (AC) outputs, so we need to transform the AC signals into the equal DC signals.

\subsection{Body Activity Detection Mode Signal Processing}

This mode is opposite to the body stress-reaction mode, which means it detects the body activity turning from the

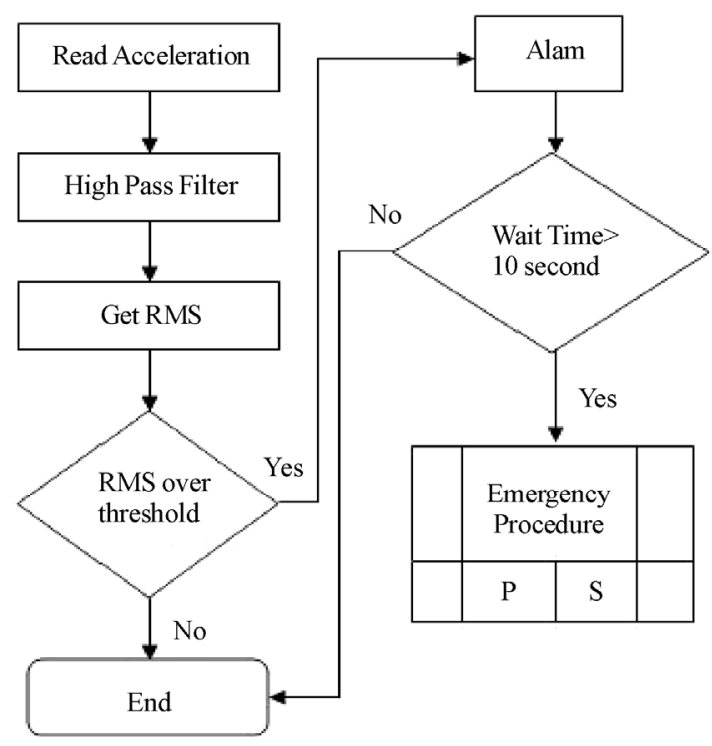

Figure 10. Body stress-reaction mode procedure flow chat. 
normal level to a low activity level. It represent something wrong might happened as well [19], and BAD starts the emergency procedures immediately. For example if the user passes out while exercising or if there is no move reaction while working. Figure 11 presents the body activity detection mode procedure flow chart.

Microprocessor reads the signal from accelerometer every-10 milliseconds, and pass the signal through one order passive HPF (High Pass Filter). Refer to Eq.1 [19], and then calculate the RMS (Root Mean Square). Refer to Eq.2, if the RMS is lower than the preset static RMS threshold for a period of time, it means that emergency has occurred. The system would beep out the warning sound in the first place and if the user does not turn off the alarm, the system would start emergency procedure.

\subsection{Emergency Mode Signal Processing}

When BAD determines there is a unusual condition, it would beep out the warning sound in the first place to inform the user there is a problem detected, and if the user-does not turn off the alarm, BAD would deliver the ATD dialing command to the mobile phone through Bluetooth HFP [5], and mobile phone would build up a voice connection with the terminal. While remote party answered call active, BAD deliver the emergency signals with sphygmomanometer, pulsimeter and GPS informa-

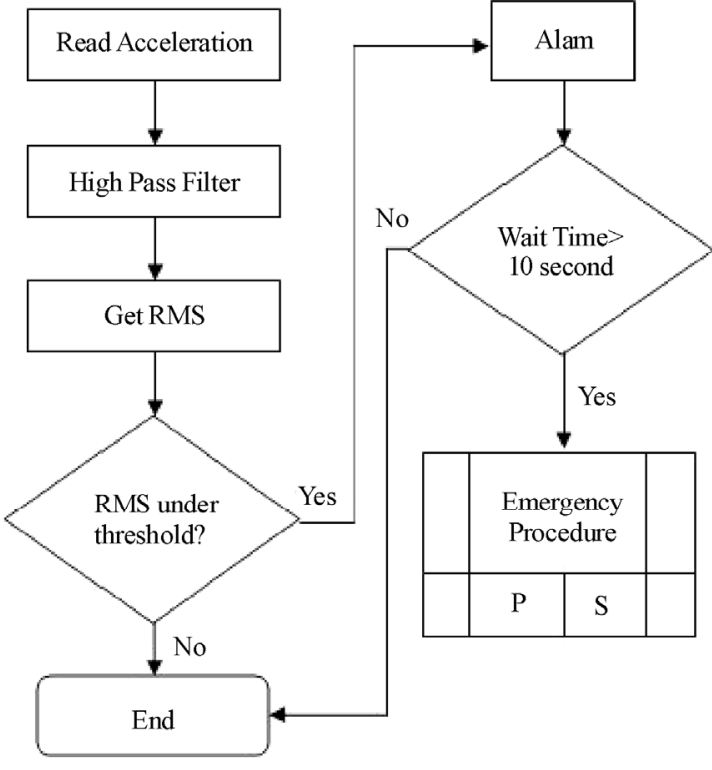

Figure 11. The body activity detection mode procedure flow chat.

tion to the monitoring server via HFP by DTMF [5] till the server is disconnected. Figure $\mathbf{1 2}$ presents the signal process of emergency procedure.

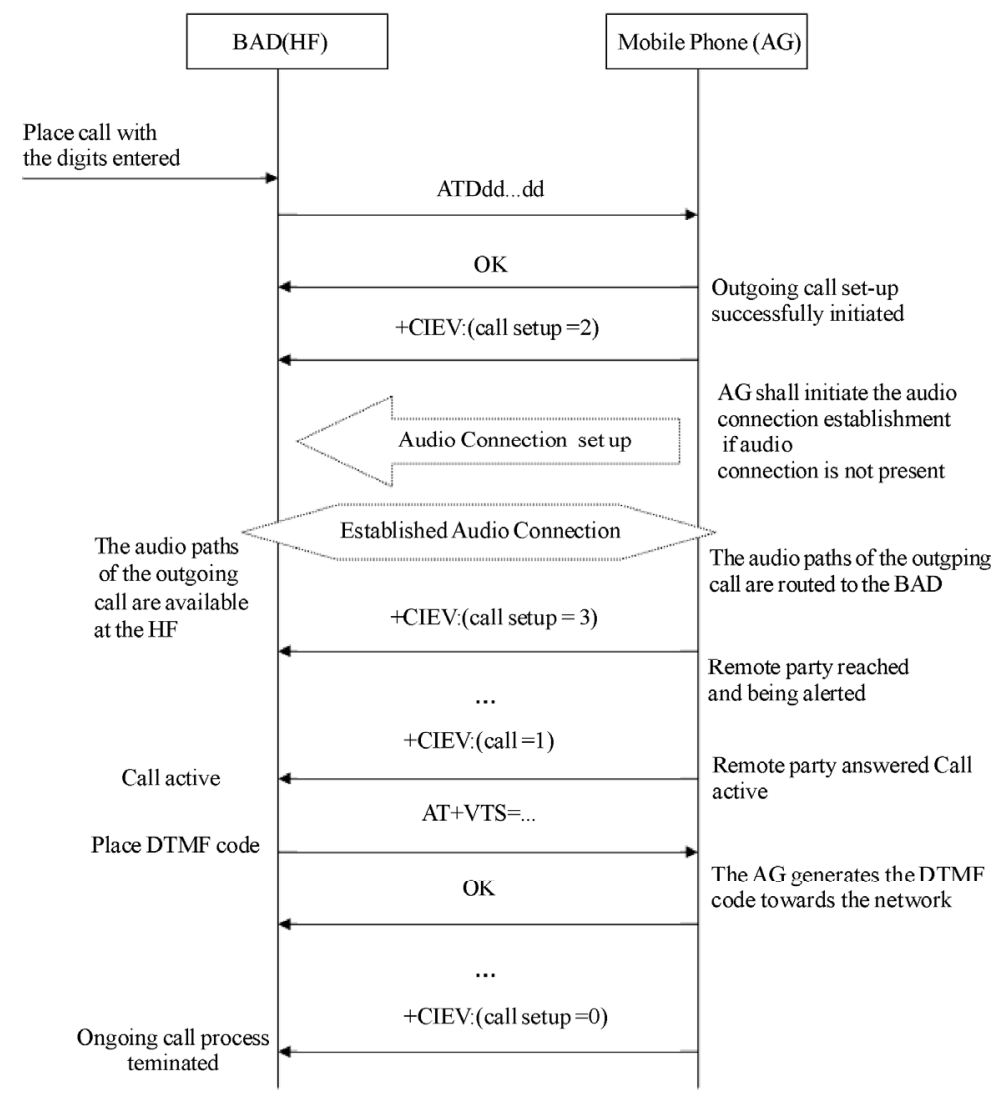

Figure 12. The signal processing of emergency procedure. 


\section{CONCLUSIONS}

This research provides a simple, practical, and portable device of real-timely monitoring human body activity to design a BAD with sphygmomanometer, pulsimeter and GPS devices, combining mobile phone technology. These devices detecting user being in both dynamic and static emergency by body stress-reaction mode and body activity detection mode separately, and suggests a solution in the emergency situation. Especially for senior care and patients who do face a risk, we expect people, who carry with this device, would have more effective care and help from others.

\section{REFERENCES}

[1] Cummins, R.O., Eisenberg, M.S., Hallstrom, A.P. and Litwin, P.E. (1985) Survival of out-of-hospital cardiac arrest with early initiation of cardiopulmonary resuscitation. American Journal of Emergency Medicine, 3(2), 114119.

[2] Albarede, J.L., Lemieux, A., Vellas, B. and Groulx, B. (1989) Psychological factors in falls in elderly patients. Canadian Journal of Psychiatry, 34(2), 94-96.

[3] Maki, B.E., Holliday, P.J. and Topper, A.K. (1991) Fear of falling and postural performance in the elderly. Journal of Gerontology, 46(4), 123-131.

[4] Wang, C.S., Lee, J.H. and Chu, Y.T., (2007) Mobile telemedicine application and technologies on GSM. Bioinformatics and Biomedical Engineering, ICBBE, the 1st International Conference, 6-8 July 2007, 1125-1128.

[5] Bluetooth Special Interest Group (2005) Bluetooth Specification, Hands-Free Profile (HFP) 1.5, November 25.

[6] Bluetooth Special Interest Group (2001) Bluetooth spe- cification. Serial Port Profil, 1.1, February 22.

[7] National Semiconductor Inc. (2005) SC14440/431/432, SC14435, SC14436/437/438. Baseband Processor for PP/FP DECT and WDCT, 1.1, June 16.

[8] MEMSIC Inc. (2007) MXA2500G/M Improved, Ultra Low Noise \pm 1.7 g Dual Axis Accelerometer with Absolute Outputs, Rev. G, June.

[9] CSR Inc. (2006) BlueCore3-ROM CSP Product Data Book, November.

[10] SiRF Inc. (2003) SiRF starIIe/LP Chip Set Data Sheet, Rev 1.1, February.

[11] Nissila, S., Sorvisto, M., Sorvoja, H., Vieri-Gashi, E. and Myllyla, R. (1998) Non-invasive blood pressure measurement based on the electronic palpation method. Engineering in Medicine and Biology Society, Proceedings of the 20th Annual International Conference of the IEEE, 4, 1723-1726.

[12] Y.-B. Lin and I. Chlamtac, (2000) Wireless and mobile network architectures. Wiley Computer Publishing, New Delhi.

[13] Wang, C.-S. (2010) Mobile and wireless technologies applying on sphygmomanometer and pulsimeter for patients with pacemaker implementation and other cardiovascular complications. Journal of Biomedical Science and Engineering, 3(1), 47-51.

[14] HoneyWell, (2002) SSC Series Pressure Sensors 0-5 psi Through 0-300 psi.

[15] Winbond Inc., (2006) W681388 Single Programmable Extended CODEC/SLIC, Ver. $\mathbf{1 . 0}$.

[16] Micrpchip Inc., (2007) PIC24FJ64GA004 Family Data Sheet.

[17] Bluetooth Special Interest Group (2004) Specification of the Bluetooth System Core, Ver., 2.0.

[18] Pravin Bhagwat Reefedge Inc., (2001) Bluetooth: Technology for short-range wireless apps, IEEE Internet Computing, 5, 96-103.

[19] MEMSIC Inc., (2005) Body Activity Detection Reference Design, August 25. 